\title{
42nd ANNUAL SASKATCHEWAN CHRISTMAS BIRD COUNT - 1983
}

Compiled by MARY I. HOUSTON, 863 University Drive, Saskatoon, Saskatchewan. S7N $0 J 8$

The intrepid group of bird counters which has descended upon Fort Walsh regardless of weather, each year since 1976, was on hand again this year, braving $-30^{\circ} \mathrm{C}$ to $-20^{\circ} \mathrm{C}$ temperatures with winds up to $60 \mathrm{~km} / \mathrm{hr}$. To top it off, they counted the highest number of species for Saskatchewan, with 36 species seen on count day and 4 additional species seen during count period, but not on count day. The second highest count was taken at Big Gully Creek, where 30 species were seen on count day with 4 additionals during the count period. Fort Walsh counters also turned up the only new species for the all time Saskatchewan Christmas Bird Count list - a Belted Kingfisher. This brings the all time total of count day species to 137 plus 2 races and 2 additionals.

Counters reported from 62 localities - a good number despite the severe weather before Christmas and the freezing rain at New Year's. Sixty-six species on count day with 4 additionals during count period is less than usual, perhaps because of the spells of poor weather.

No Great Gray Owls or Pine Siskins were reported this year. Bohemian Waxwings made a comeback, and there were fewer Cedar Waxwings last year's increase in numbers and range has not continued. There was a marked increase in the number of localities reporting Merlins - 14 in 62 counts in 1983 as compared to 8 in 63 counts in 1982. Probably the most striking increase was in the number and range of Rosy Finches. First reported in 1945 and 1946 at Dollard, they were not reported again until 1975 when 1 was seen at Spring Valley. At Fort Walsh in 1976, 140 were seen; in 1977, 23; in 1978, 1, in 1979, 22. At Eastend 65 were seen in 1980. In 1981 and 1982 Rosy Finches were not reported in any count. This year, however, they were reported from 5 different areas: Biggar (1), Fort Walsh (488), Govenlock (3), Swift Current (25), and White Bear (25).

After the list of participants from the 62 localities, and the map, Table 1 gives the Coverage and the Weather; Table 2 gives species reported in more than 3 locations and gives numbers of each species seen in each locality on count day, with numbers for additional species during count period indicated with a + sign; Table 3 gives numbers and locations of species seen in 3 or fewer locations during count period. Table 4 gives locations and numbers of birds not positively identified as to species.

\section{Count Areas and Participants}

Names of compilers in italics.

1. ASSINIBOIA. Ed. Bearss, Elaine Brunt, Jack Burgeson, Cec Hayward, Don Lemond, Ted McMorrine, Wilf Prentice, Ken Schuweiler.

2. BANGOR. Mrs. Katie Thompson.

3. BIGGAR. Kelly Foster, Harvey Renaud, Wayne Renaud, Guy Wapple, Robert Wapple.

4. BIG GULLY CREEK. Wayne 
Harris, Sheila Lamont.

5. BIRCH HILLS. Terry Toews, Donald A. Weidl.

6. BRIGHTWATER RESERVOIR. Mary Gilliland, Cliff Matthews, Alan R. Smith.

7. BROADVIEW. Dave J. Chaskavich, Don Weidl.

8. CROOKED LAKE. A Weidl, Donald A. Weidl.

9. DILKE. Margaret Belcher, Bertha Wilson.
10. DUCK MOUNTAIN PARK. Bill Anaka, Joyce Anaka, Ben Kruser, JoAnn Radish.

11. DUVAL. J. Rawdon Bieber, Tony Lang, lain Richardson, Lloyd Saul, Catherine M. Schmidt.

12. EASTEND. Bart and Bruce Lebastard, Henri Lebastard.

13. ENDEAVOUR. Norman Harris.

14. FORT QU'APPELLE. Manley Callin, Errol Cochrane, Ethel Cockwill, Bernie de Vries, David and Ron Hooper, Alice Laing, Vic

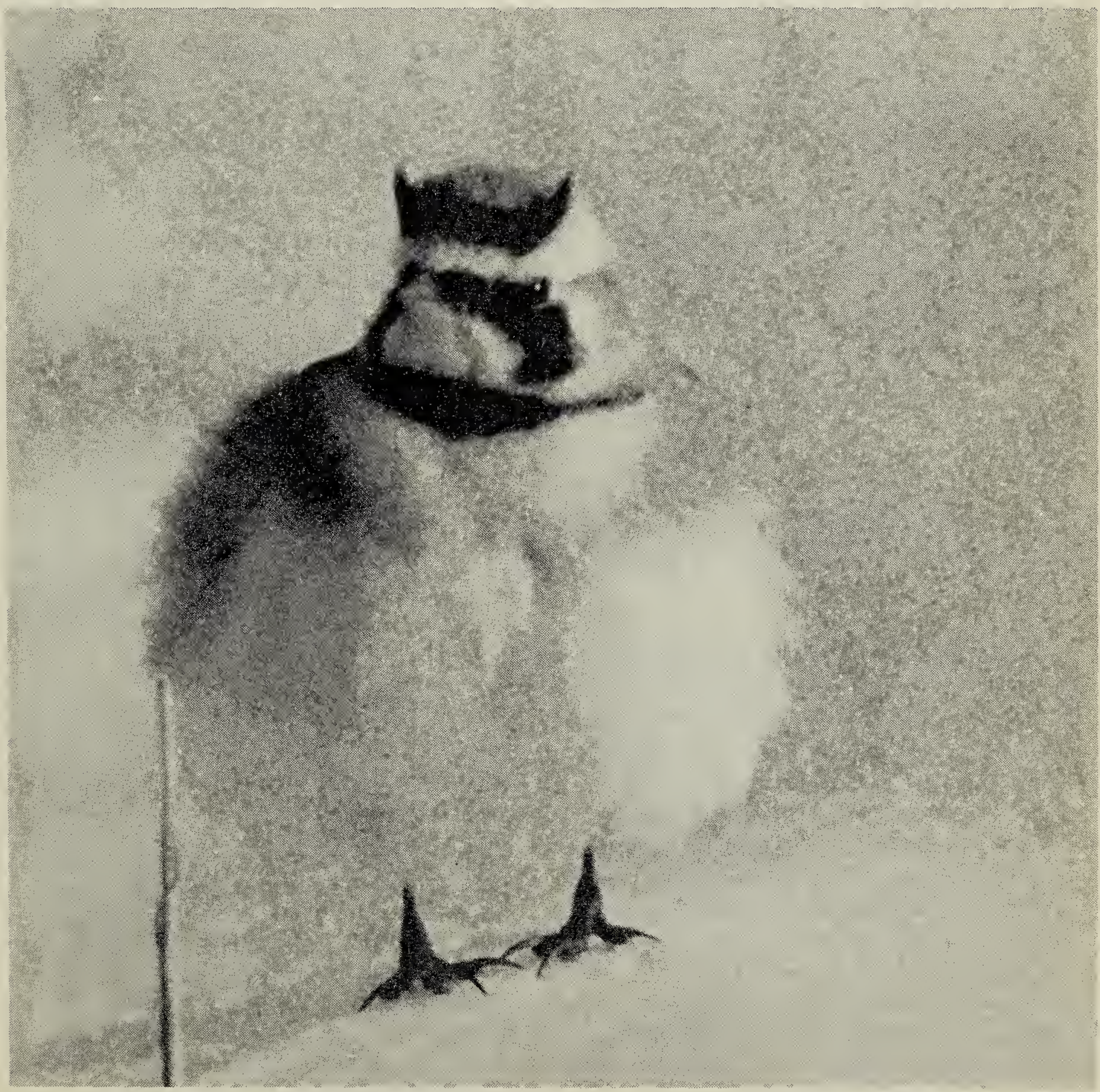


and Lois Lamontagne, Ray Mlazgar, Dick and Sonia Morrell, James Peigan, Lorne and Shawn Rowell, Bruno Skolski.

15. FORT WALSH. Chris Escott, Wayne Harris, Sheila Lamont, Wilkes Parsonage, Wayne Renaud, Guy Wapple, Robert Wapple.

16. GARDINER DAM. Wayne Harris, Sheila Lamont, Wayne Renaud, Guy Wapple, Robert Wapple.

17. GLAMIS-WISETON. Brian $H$. Jones, G.L. Jones.

18. GOODSOIL. Dr. and Mrs. D. N. Morton, Richard Morton.

19. GOOD SPIRIT LAKE. Bill Anaka, Joyce Anaka.

20. GOVENLOCK. Wayne Harris, Sheila Lamont, Wayne Renaud, Guy Wapple, Robert Wapple.

21. GRAND CENTRE (Sask-Alta). J. F. Roy, Robin and Ryan Roy.

22. GREENWATER LAKE. Glen $O$. Honig.

23. HUMBOLDT. Ed Brockmeyer, Ev and Warren Hoffman, Dwayne Saretsky.

24. INDIAN HEAD. Cecil and Betty Ashmore, Jan Beattie, Yvonne Brown, Raymond Crawford, Jim Elliott, Richard Escott, Roger Gray, Mavis Gray, Joan Halford, Gordon Howe, Janet LePrieur, Eric Peterson, Joan and Lorne Scott, Gertie and Reg Scott, Fred Skinner, Donna and Ron Thompson and family, Anne and Gordon Willerth.

25. KELVINGTON. Chris Donovan, James Donovan.

26. KENASTON. P. Lawrence Beckie.
27. KINDERSLEY. Jean M. Harris. Keith Harris.

28. KUTAWAGAN LAKE. Wayne Harris, Sheila Lamont.

29. LA RONGE. Jim Paul.

30. LAST MOUNTAIN LAKE. Wayne Harris, Sheila Lamont.

31 LEADER. Daisy D. Meyers.

32. LOON LAKE. A Davis, P. Davis, J. Huys, M. Huys, Frank Scott.

33. LOVE-TORCH RIVER. Bert Dalziel, Joan Dalziel, Betty, Bruce, Sean and Troy Donovan, Elwood and Pearl French, Bill and Lynn Mathews, Bill Rode.

34. LUSELAND. Kim Finley, Estelle Martfeld, Bev and Brent Honeker.

35. MAIDSTONE BRIDGE. Wayne Harris, Sheila Lamont.

36. MELFORT. Philip Curry, Glenn Galloway, Ted Morton, John Waddington, Ron Zdan.

37. MOOSE JAW. Ben and Isabelle Cruise, Alice and Carl Ellis, Doug Francis, Alan, Arthur, Hartley and Edward Fredeen, Edith and Pat Kern, Eve King, Cy Knight, Leith Knight, Bernice and Moray Lewis, Arie and Henry Van Dorland, Sheina Wait.

38. NIPAWIN. Bert and Joan Dalziel, Mr. and Mrs. Fred Diener, Bruce Donovan.

39. NUT MOUNTAIN. Chester Sloan, Dianne Sloan, Marguerite Sloan.

40. PELICAN NARROWS. Gabrielle Hembry, Gordon Speirs.

41. PIKE LAKE. Margaret duChalard, Chris Escott, Mary Gilliland, J. B. Gollop, Jane and Mike Gollop, 
Frank Roy, Stan Shadick, Jim Wedgewood.

42. PRINCE ALBERT. Pamela and John Burt.

43. PRINCE ALBERT NATIONAL PARK. Cam Alexander, Doug Anions, Chris Doupe, Greg Keesey, Bradley Muir, June-Ann Muir, Kathy Ryhorchuk, Pat Strutt, Jean and Mervyn Syroteuk, Andrew Whiting.

44. RAYMORE. Wayne Harris, Sheila Lamont.

45. RED DEER HILL. Kevin Aschim, Kim Hruska.

46. REGINA. Chris Adam, Margaret Belcher, Tom Beveridge, Lucille Bradatsch, Frank Brazier, Phil Browne, Paul Chytyk, Kathleen Donaver, Paule Hjertaas, Robert Kreba, Tony Lang, Bob Luterbach, Christine MacDonald, Jim Materi, John McDonald, Ron Myers, Chuck Newyar, Irene Pierson, Brian Rainey, Sarogini Ramnarine, Tom Riffel, Frank Switzer, Ian Switzer, Dorothy Tegart, John Triffo, Christophe Wilhelm, Pierre Wilhelm.

47. ROUND LAKE. Doug Francis, Joe Grimeau, Pat Kern, Les Tremblay.

48. ST. WALBURG. Nigel Caulkett.

49. SASKATOON. Michele Anderson, Juhachi Asai, Eric Densem, Anne Dzus, Chris Escott, Grant Forsythe, Charles, John, Jon, Naomi, Pauline and Tom Gerrard, Mary Gilliland, Bernie Gollop, John Hanbidge, Simon Heath, Mary Houston, Stuart Houston, Blake Maybank, Don and Jo McRobbie, Betty and Jim Mundy, Bill Nickel, Jim and Pat O'Neil, Evelyn Paterson, John Polson, Charlie Simpson, Alan Smith, Bruce
Tomlinson, Jim and Shirley Wedgwood, David Wright, Kelly Wylie.

50. SCOTT. Wayne Renaud, Guy Wapple, Robert Wapple.

51. SKULL CREEK. J. Bennetto, Michelle Schuler.

52. SPRING VALLEY. Allan, Larry \& Nick Bogdan, Flossie Bogdan, Ernie Jordison.

53. SQUAW RAPIDS. Wayne Harris, Sheila Lamont, Wayne Renaud, Guy Wapple, Robert Wapple.

54. SOMME. Margaret and Stan Back, Alice, Edwin and Shirley Billeter, Ian and Myrla Birch, David and Wesley Black, Florence Chase, Donald Hooper.

55. SWIFT CURRENT. Doug Adams, Catherine Dumouchel, Stan Greenwood, Christopher Harris, Earle Hayes, Jack Ricou, Russ Wall.

56. TISDALE. Joyce Mohr.

57. TONKIN. Ben Kruser, JoAnn Radish.

58. WAUCHOPE. Dale Hjertaas, Paule Hjertaas.

59. WHITE BEAR. Bruce Brantley, Lynette Jordheim, Sig Jordheim.

60. WHITEBEECH. Ida Wotherspoon, Lindsay Wotherspoon, Ed Terlesky.

61. WOLSELEY SOUTH. Dale Chay, Donald Hayward.

62. YORKTON. Joyce Anaka, Bill Anaka, Joyce Baines, Walter Baines, Joe Bergerman, Warren Hjertaas, Lance Irvine, Ben Kruser, JoAnn Radish, Birkert Satterthwaite, Harold Wilkinson. 
1. ASSINIBOIA

2. BANGOR

3. BIGGAR

4. BIG GULLY CREEK

5. BIRCH HILLS

6. BRIGHTWATER RESERVOIR

7. BROADVIEW

8. CROOKED LAKE

9. DILKE

10. DUCK MOUNTAIN PARK

11. DUVAL

12. EASTEND

13. ENDEAVOUR

14. FORT QU'APPELLE

15. FORT WALSH

16. GARDINER DAM

17. GLAMIS-WISETON

18. GOODSOIL

19. GOOD SPIRIT LAKE

20. GOVENLOCK

21. GRAND CENTRE (Sask.-Alta.)

22. GREENWATER LAKE

23. HUMBOLDT

24. INDIAN HEAD

25. KELVINGTON

26. KENASTON

27. KINDERSLEY

28. KUTAWAGAN LAKE

29. LA RONGE

30. LAST MOUNTAIN LAKE

31. LEADER
32. LOON LAKE

33. LOVE-TORCH RIVER

34. LUSELAND

35. MAIDSTONE BRIDGE

36. MELFORT

37. MOOSE JAW

38. NIPAWIN

39. NUT MOUNTAIN

40. PELICAN NARROWS

41. PIKE LAKE

42. PRINCE ALBERT

43. PRINCE ALBERT NATIONAL PARK

44. RAYMORE

45. RED DEER HILL

46. REGINA

47. ROUND LAKE

48. ST. WALBURG

49. SASKATOON

50. SCOTT

51. SKULL CREEK

52. SPRING VALLEY

53. SQUAW RAPIDS

54. SOMME

55. SWIFT CURRENT

56. TISDALE

57. TONKIN

58. WAUCHOPE

59. WHITE BEAR

60. WHITEBEECH

61. WOLSELEY SOUTH

62. YORKTON

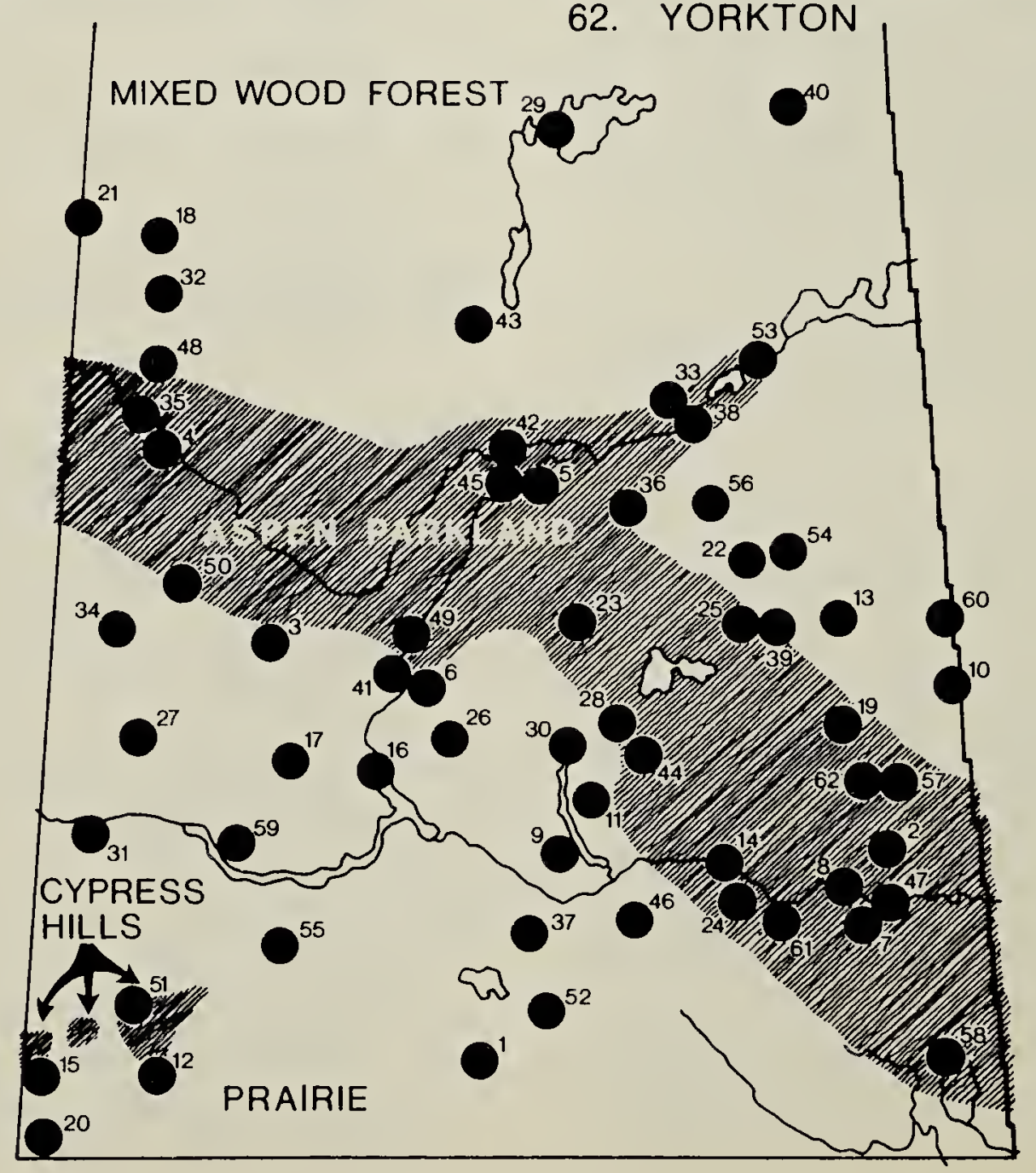


Table 1. CHRISTMAS COUNT COVERAGE AND WEATHER CONDITIONS

\begin{tabular}{|c|c|c|c|c|c|c|c|c|c|}
\hline 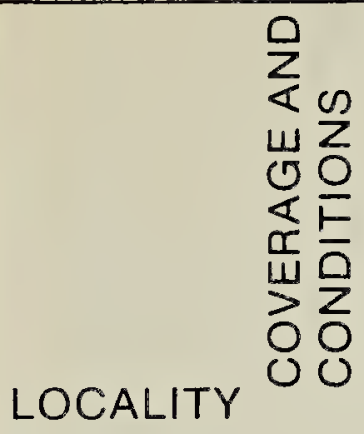 & 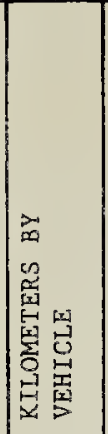 & 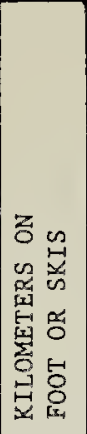 & 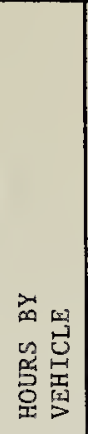 & 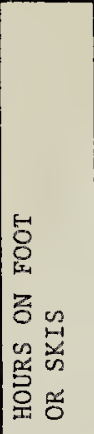 & 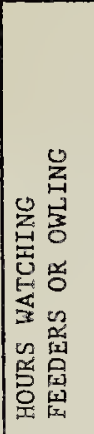 & 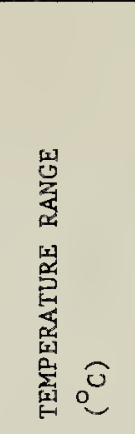 & 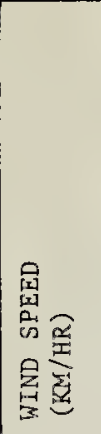 & 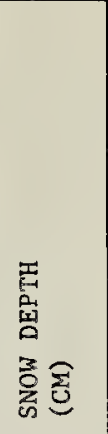 & 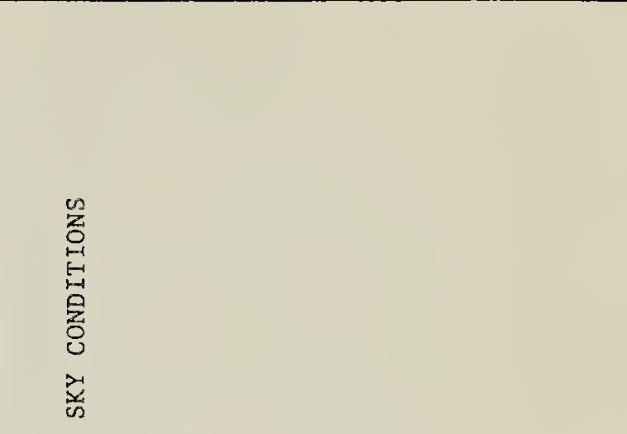 \\
\hline \multirow{5}{*}{$\begin{array}{l}\text { ASSINIBOIA } \\
\text { BANGOR } \\
\text { BIGGAR } \\
\text { BIG GULLY CREEK } \\
\text { BIRCH HILLS }\end{array}$} & 60 & - & 2 & - & - & $-2 /+2$ & $10-20$ & 13 & mostly clear; clear \\
\hline & 26 & $=$ & 0.5 & $=$ & 1 & $-30 /-18$ & $20-25$ & $15-20$ & partly cloudy \\
\hline & 183 & 17 & 11 & 6.5 & - & $-38 /-33$ & $13-20$ & $15-25$ & mostly clear; clouding \\
\hline & 130 & 21 & $\frac{5}{5}$ & 7 & - & $-19 /-5$ & $5-15$ & $30-35$ & clear; partly cloudy \\
\hline & 144 & - & 5 & - & 3 & $-36 /-33$ & $0-5$ & $20-30$ & clear \\
\hline \multirow{5}{*}{$\begin{array}{l}\text { BRIGHTWATER RESERVOIR } \\
\text { BROADVIEW } \\
\text { CROOKED LAKE } \\
\text { DILKE } \\
\text { DUCK MOUNTAIN PARK }\end{array}$} & 80 & 5 & 8 & 4 & - & -30 & $0-5$ & $5-20$ & mostly clear \\
\hline & 119 & 9 & $\frac{4}{4}$ & 3 & - & $-22 /-19$ & $20-15$ & $10-15$ & partly cloudy \\
\hline & 83 & 2 & $\frac{1}{4}$ & 1 & - & $-117-9$ & $10-15$ & $20-30$ & overcast, light snow; cloudy \\
\hline & 100 & \begin{tabular}{|c|c|c|c|}
3 \\
\end{tabular} & 2.5 & 1 & 3 & $-7 /-2$ & 10 & $0-10$ & clear \\
\hline & 22 & 22 & 0.5 & 5.5 & - & $-4 / 0$ & $15-20$ & 20 & light overcast \\
\hline \multirow{5}{*}{$\begin{array}{l}\text { DUVAL } \\
\text { EASTEND } \\
\text { ENDEAVOUR } \\
\text { FORT QU'APPELLE } \\
\text { FORT WALSH }\end{array}$} & 205.5 & 10 & 7.5 & 3 & - & $-13 /-18$ & $4-16$ & $4-30$ & clear \\
\hline & 15 & $\frac{2}{2}$ & 4 & 1.5 & - & -13 & & 12 & \\
\hline & 23 & $\frac{2}{3}$ & 3 & 2 & - & $-22 /-12$ & $5-10$ & $20-25$ & partly cloudy; overcast \\
\hline & 200 & - & 10 & - & 3 & -12 & 27 & 13 & \\
\hline & 90 & 41 & 8.5 & 20.5 & 1 & $-30 /-20$ & $0-60$ & $10-40$ & clear; partly cloudy \\
\hline \multirow{5}{*}{$\begin{array}{l}\text { GARDINER DAM } \\
\text { GLAMIS - WISETON } \\
\text { GOODSOIL } \\
\text { GOOD SPIRIT LAKE } \\
\text { GOVENLOCK }\end{array}$} & 169 & 12 & 13 & 4 & - & $-36 /-25$ & $0-10$ & $10-20$ & clear \\
\hline & $\frac{15}{55}$ & 3.5 & 2.5 & $\frac{7}{1}$ & $=$ & $-10 /-15$ & $10-20$ & $12-20$ & mostly clear \\
\hline & 25 & - & 3 & - & 6 & $-7 / 0$ & $0-5$ & $15-20$ & clear \\
\hline & 90 & 2.5 & 4 & 0.5 & 1 & $-1.7 /-19$ & $30-40$ & 17 & mostly clear \\
\hline & 110 & 9 & 10.5 & 5.5 & - & $-34 /-20$ & $0-30$ & $1-10$ & clear; partly cloudy \\
\hline \multirow{5}{*}{$\begin{array}{l}\text { GRAND CENTRE } \\
\text { GREENWAIER LAKE } \\
\text { HUMBOI,DT } \\
\text { INDIAN HEAD } \\
\text { KELVINGTON }\end{array}$} & 130 & 1 & 4 & 1 & - & $-12 /-13$ & $19-24$ & $25-30$ & overcast \\
\hline & - & 5 & - & 2 & 5 & $-15 /-5$ & 0 & $20-25$ & mostly clear \\
\hline & 15 & 8 & 0.5 & 2 & 4 & $-10 /-12$ & $5-10$ & $18-20$ & mostly clear \\
\hline & 32 & 5 & 2 & 1 & 6 & $-16 /-18$ & $20-25$ & $20-24$ & mostly clear; partly cloudy \\
\hline & 10 & - & - & - & 4 & $-10 /-5$ & 0 & $18-24$ & clear \\
\hline \multirow{10}{*}{$\begin{array}{l}\text { KENASTON } \\
\text { KINDERSLEY } \\
\text { KUTAWACAN LAKE } \\
\text { LA RONGE } \\
\text { LAST MOUNTAIN LAKE } \\
\text { LEADER } \\
\text { LOON LAKE } \\
\text { LOVE - TORCH RIVER } \\
\text { LUSELAND } \\
\text { MAIDSTONE BRIDGE }\end{array}$} & 100 & 5 & 4 & 2 & - & $-15 /-10$ & 10 & 20 & mostly clear \\
\hline & 26 & - & 2 & - & - & & & & overcast \\
\hline & 250 & 3 & 7 & 2 & - & $-41 /-37$ & $8-40$ & $5-25$ & clear \\
\hline & 48 & 9 & 3 & 2 & 0.5 & $-17 /-8$ & $10 \sim 18$ & $20-30$ & mostly clear; partly cloudy \\
\hline & 180 & 3 & 6 & 2 & - & $-5 /+3$ & $5-30$ & $6-8$ & clear; partly cloudy \\
\hline & 4 & 3 & 1 & 2 & - & $-1 /+5$ & $10-20$ & $8-16$ & partly cloudy \\
\hline & 15 & 2 & 0.5 & 1 & 3 & $0 /-5$ & 5 & 30 & overcast, light rain \\
\hline & 100 & - & 4 & - & 6 & $-25 /-14$ & $0-5$ & $20-25$ & partly cloudy; overcast \\
\hline & 43 & 10 & 1.5 & 4 & - & $-20 / \cdots 25$ & $10-15$ & $6-13$ & most1y clear; clear \\
\hline & 130 & 5 & 6 & 2 & - & $-15 /-5$ & $0-10$ & $30-35$ & partly cloudy \\
\hline \multirow{5}{*}{$\begin{array}{l}\text { MELFORT } \\
\text { MOOSE JAW } \\
\text { NIPAWIN } \\
\text { NUT MOUNTAIN } \\
\text { PELICAN NARROWS }\end{array}$} & 20 & 3 & 1 & 3 & 1 & $-15 /-10$ & 0 & $20-30$ & clear \\
\hline & 196 & 15 & 9.5 & 7.5 & 1 & $-13 /-15$ & $15-20$ & $7-15$ & overcast \\
\hline & -40 & - & 2 & - & 2 & $-29 /-24$ & $0-5$ & $30-35$ & clear \\
\hline & 10 & 1 & 0.5 & 0.5 & 8 & $-16 /-13$ & 5 & $21-26$ & clear; mostly cloudy \\
\hline & 30 & 3 & 2 & 1 & - & $-15 /-20$ & $0-10$ & $10-20$ & clear; mostly clear \\
\hline \multirow{5}{*}{$\begin{array}{l}\text { PIKE LAKE } \\
\text { PRINCE ALBERT } \\
\text { P.A. NATIONAL PARK } \\
\text { RAYMORE } \\
\text { RED DEER HILL }\end{array}$} & 148 & 16 & 12 & 11 & - & $-9 / 0$ & $0-15$ & $10-20$ & partly cloudy \\
\hline & - & - & - & & 10 & $-10 / 5$ & $5-10$ & $10 \sim 25$ & mostly cloudy, rain; mostly clear \\
\hline & 152 & 20 & 9.5 & 12.5 & 13.5 & $-25 /-31$ & $10-15$ & $15-25$ & partly cloudy; clear \\
\hline & 235 & 5 & 8 & 3 & - & $-38 /-27$ & $5-25$ & $25-30$ & clear; becoming partly cloudy \\
\hline & 90 & 14 & 3 & 3.5 & - & $-10 /-15$ & $5-35$ & $10-15$ & clear \\
\hline \multirow{5}{*}{$\begin{array}{l}\text { REGINA } \\
\text { ROUND LAKE } \\
\text { ST. WALBURG } \\
\text { SASKATOON } \\
\text { SCOTI }\end{array}$} & 427 & 77 & 23 & 23 & - & $-13 /-10$ & $20-26$ & $15-30$ & Dartly cloudy, light snow; mostly clear \\
\hline & 61 & 4.5 & 4 & 2 & 2 & $-5 /+5$ & & $10-15$ & mostly clear; partly cloudy \\
\hline & 150 & 10 & 4 & 3 & - & $-10 /-15$ & $\overline{10-15}$ & $15-30$ & mostly cloudy \\
\hline & 320 & $\frac{1}{47}$ & 36 & 33 & 16 & $-14 /-11$ & $11-19$ & $12-18$ & overcast \\
\hline & 245 & 11 & 7 & 5.5 & - & $-19 /-9$ & $3-10$ & $10-20$ & partly cloudy; mostly clear \\
\hline \multirow{5}{*}{$\begin{array}{l}\text { SKULI CREEK } \\
\text { SPRING VALLEY } \\
\text { SQUAW RAPIDS } \\
\text { SOMME } \\
\text { SWIFT CURRENT }\end{array}$} & 16 & 6 & 2 & 2 & 0.5 & $-18 /-15$ & $25-30$ & 30 & overcast \\
\hline & 122 & 10 & 5 & $-\frac{2}{2}$ & - & $-2 /+1$ & $15-25$ & $10-15$ & most $1 y$ clear \\
\hline & 100 & $\frac{16}{16}$ & 13 & $\frac{2}{7}$ & - & $-37 /-20$ & $5-35$ & $\frac{8-10}{10}$ & overcast; intermittent freezlng rain \\
\hline & 330 & 3 & 9 & 2 & 5 & $-4 /-3$ & $0-5$ & $20-30$ & \\
\hline & 307 & 21 & 11.5 & 9.5 & - & $-35 /-25$ & $30-40$ & $5-75$ & clear \\
\hline \multirow{5}{*}{$\begin{array}{l}\text { TISDALE } \\
\text { TONKIN } \\
\text { WAUCHOPE } \\
\text { WHITE BEAR } \\
\text { WHITEBEECH }\end{array}$} & 30 & - & 1 & - & 2 & $-25 /-20$ & $10-15$ & 16 & clear: partly cloudy \\
\hline & 34 & 2 & 1.5 & 2 & - & $-22 /-24$ & $0-5$ & $2-10$ & clear \\
\hline & 46 & 6 & 1.5 & 3 & 1.5 & -10 & 15 & $15-20$ & \\
\hline & $\frac{45}{15}$ & 18 & 0.5 & $\frac{5}{6}$ & - & $-9 /-6$ & $0-1$ & $20-40$ & clear \\
\hline & 20 & 2 & 1 & 1 & 6 & $-4 /-8$ & $0-5$ & $14-16$ & clear \\
\hline \multirow{2}{*}{$\begin{array}{l}\text { WOLSELEY SOUTH } \\
\text { YORKTON }\end{array}$} & 62 & 1 & 2 & 0.5 & _ & -5 & 0 & $9-11$ & clear \\
\hline & 155 & 4 & $\frac{2}{2}$ & 2 & - & -23 & 8 & 10 & clear; partly cloudy \\
\hline
\end{tabular}




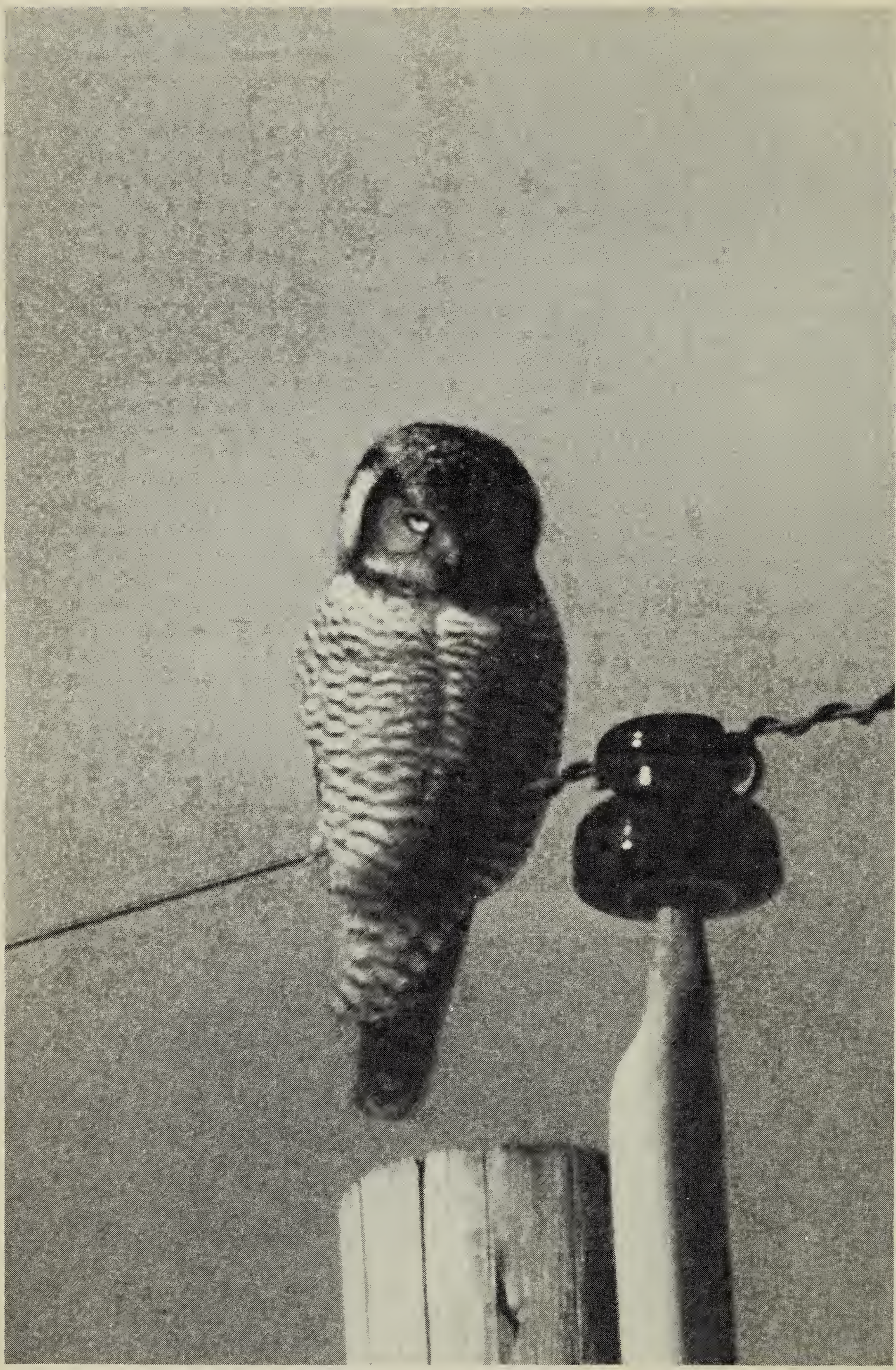


Table 2-1. SPECIES RECORDED FROM MORE THAN THREE LOCALITIES. A PLUS SIGN INDICATES A HIGH COUNT FOR A SPECIES SEEN DURING COUNT PERIOD BUT NOT ON COUNT DAY.

\begin{tabular}{|c|c|c|c|c|c|c|c|c|c|c|}
\hline $\begin{array}{l}1 \\
\overline{1} \\
\vdots \\
0 \\
0\end{array}$ & 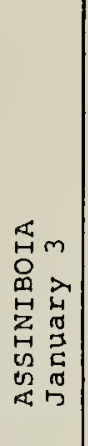 & & 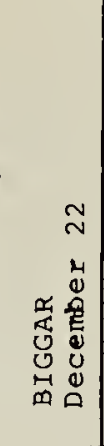 & 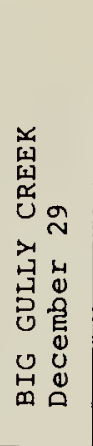 & 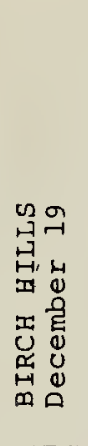 & 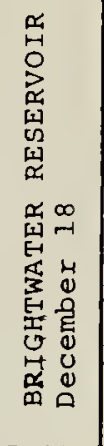 & 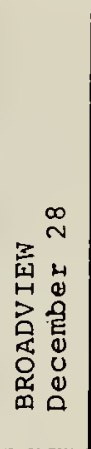 & 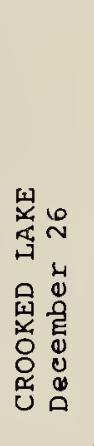 & 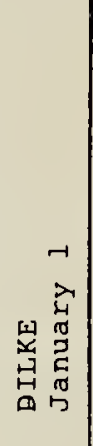 & 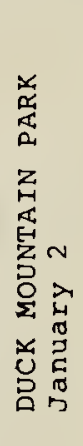 \\
\hline \multirow{5}{*}{$\begin{array}{l}\text { MALLARD } \\
\text { COMMON GOLDENEYE } \\
\text { BALD EAGLE } \\
\text { NORTHERN GOSHAWK } \\
\text { GOLDEN EAGLE } \\
\end{array}$} & & & 1 & & & & & 1 & & \\
\hline & & & & & & & & & & \\
\hline & & & & 1 & & & & & & \\
\hline & & & 1 & 2 & & & & & & \\
\hline & & & & & & & & & & \\
\hline \multirow{5}{*}{$\begin{array}{l}\text { MERLIN } \\
\text { GRAY PARTRIDGE } \\
\text { RING-NECKED PHEASANT } \\
\text { RUFFED GROUSE } \\
\text { SHARP-TAILFN GROUSE }\end{array}$} & & & 1 & 1 & & 1 & & & & \\
\hline & 50 & & 10 & +7 & & 12 & 8 & & & \\
\hline & 7 & & & +1 & & & 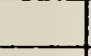 & & & \\
\hline & & & & 1 & & & 1 & & & \\
\hline & 6 & & 43 & 12 & & 2 & 5 & 99 & 23 & \\
\hline \multirow{5}{*}{$\begin{array}{l}\text { ROCK DOVE } \\
\text { GREAT HORNED OWL } \\
\text { SNOWY OWL } \\
\text { DOWNY WOODPECKER } \\
\text { HAIRY WOODPECKER }\end{array}$} & 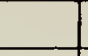 & & 154 & 77 & 55 & 27 & 9 & 2 & 12 & \\
\hline & 2 & & 2 & 2 & 2 & 2 & 1 & 1 & 2 & \\
\hline & 2 & & +1 & +1 & 1 & & & & $\ldots$ & \\
\hline & & 1 & 1 & 2 & 1 & 1 & 1 & & +1 & 1 \\
\hline & & 1 & & 4 & 1 & 1 & 2 & 1 & & \\
\hline \multicolumn{11}{|l|}{ THREE-TOED WOODPECKER } \\
\hline \multirow{4}{*}{$\begin{array}{l}\text { BLACK-BACKED WOODPECKER } \\
\text { NORTHERN FLICKER } \\
\text { PILEATED WOODPECKER } \\
\text { HORNED LARK }\end{array}$} & & & & 1 & & & & & & \\
\hline & & & & & & & & & & \\
\hline & & & & +1 & & & & & & \\
\hline & & & 40 & 2 & & & & & +7 & \\
\hline \multirow{5}{*}{$\begin{array}{l}\text { GRAY JAY } \\
\text { BLUE JAY } \\
\text { BLACK-BILLED MAGPIE } \\
\text { COMMON RAVEN } \\
\text { BLACK CAPPED CHICKADEE }\end{array}$} & & & & 1 & & & & & & \\
\hline & & & & 2 & +1 & & 2 & 1 & & 1 \\
\hline & 20 & 1 & 58 & 39 & 13 & 23 & 16 & 8 & 19 & \\
\hline & & & 2 & 9 & 2 & & & & & 5 \\
\hline & & 3 & 17 & 28 & 6 & 10 & 9 & 11 & 2 & 5 \\
\hline \multirow{5}{*}{$\begin{array}{l}\text { BOREAL CHICKADEE } \\
\text { RED-BREASTED NUTHATCH } \\
\text { WHITE-BREASTED NUTHATCH } \\
\text { AMERICAN ROBIN } \\
\text { BOHEMIAN WAXWING }\end{array}$} & & & 1 & 1 & & & & & & \\
\hline & & & & & & & & & & \\
\hline & & & & & & & & 1 & & \\
\hline & & & & & & & & & & \\
\hline & 2 & & 21 & 21 & 19 & 2 & 5 & 4 & & \\
\hline \multirow{5}{*}{$\begin{array}{l}\text { NORTHERN SHRIKE } \\
\text { EUROPEAN STARLING } \\
\text { AMERICAN TREE SPARROW } \\
\text { DARK-EYED JUNCO } \\
\text { SNOW BUNTING } \\
\end{array}$} & & & & 1 & & & & 1 & & \\
\hline & & & 1 & & & & & & & \\
\hline & & & & & & & & & & \\
\hline & & & & & & & & & & \\
\hline & 80 & 150 & 2310 & 251 & 339 & 1040 & 255 & 400 & 100 & \\
\hline \multirow{5}{*}{$\begin{array}{l}\text { RUSTY BLACKBIRD } \\
\text { ROSY FINCH } \\
\text { PINE GROSBEAK } \\
\text { RED CROSSBILL } \\
\text { WHITE-WINGED CROSSBILL }\end{array}$} & & & & 1 & & & & & & \\
\hline & & & 1 & & & & & & & \\
\hline & & & & 7 & & & & & +4 & \\
\hline & & & & 3 & & & & & & \\
\hline & & & & 7 & & & & & & \\
\hline \multirow{4}{*}{$\begin{array}{l}\text { COMMON REDPOLL } \\
\text { HOARY REDPOLL } \\
\text { EVENING GROSBEAK } \\
\text { HOUSE SPARROW } \\
\end{array}$} & & & & 62 & & & & & & \\
\hline & & & & 2 & & & & & & \\
\hline & & & & 29 & 8 & & 2 & & & \\
\hline & 400 & 2 & 843 & 351 & 75 & 55 & 90 & & 254 & \\
\hline \multirow{4}{*}{$\begin{array}{l}\text { NO. SPECIES IN TABLE } 3 \& 4 \\
\text { NO. COUNT DAY SPECIES } \\
\text { NO. COUNT PERIOD SPECIES } \\
\text { NO. COUNT DAY INDIVIDUALS }\end{array}$} & 0 & 0 & 1 & 4 & 0 & 0 & 0 & 0 & 0 & 0 \\
\hline & 9 & 6 & 18 & 30 & 12 & 12 & 14 & 12 & 7 & 4 \\
\hline & 9 & 6 & 20 & 34 & 13 & 12 & 14 & 12 & 10 & 4 \\
\hline & 569 & 158 & 3507 & 958 & 522 & 1176 & 406 & 530 & 412 & 12 \\
\hline
\end{tabular}


Table 2-2. SPECIES RECORDED FROM MORE THAN THREE LOCALITIES. A PLUS SIGN INDICATES A HIGH COUNT FOR A SPECIES SEEN DURING COUNT PERIOD BUT NOT ON COUNT DAY.

\begin{tabular}{|c|c|c|c|c|c|c|c|c|c|c|c|}
\hline$\frac{z}{\Xi}$ & 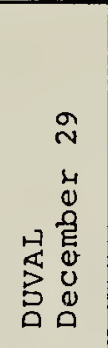 & 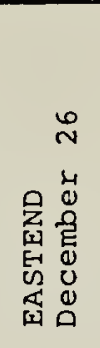 & 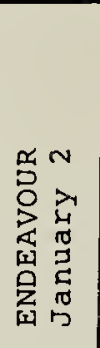 & 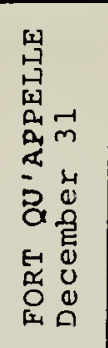 & 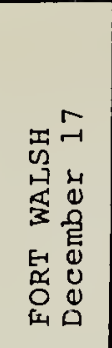 & 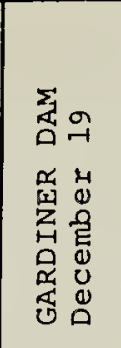 & 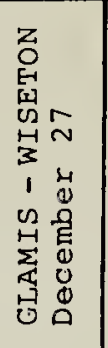 & 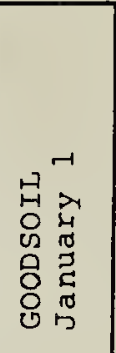 & 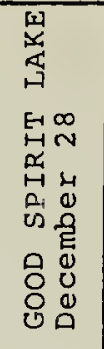 & 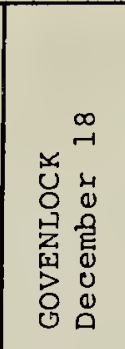 & 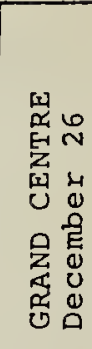 \\
\hline \multirow{5}{*}{$\begin{array}{l}\text { MALLARD } \\
\text { COMMON GOLDENEYE } \\
\text { BALD EAGLE } \\
\text { NORTHERN GOSHAWK } \\
\text { GOLDEN EAGLE } \\
\end{array}$} & & & & 7 & 3 & 346 & & & & & \\
\hline & & & & 4 & & 7 & & & & & \\
\hline & & & & +1 & +1 & 6 & & & & & \\
\hline & & & +1 & & 3 & 1 & & & & & \\
\hline & & 1 & & & 3 & & & & & 2 & \\
\hline \multirow{5}{*}{$\begin{array}{l}\text { MERLIN } \\
\text { GRAY PARTRIDGE } \\
\text { RING-NECKED PHEASANT } \\
\text { RUFFED GROUSE } \\
\text { SHARP-TAILED GROUSE } \\
\end{array}$} & & & & & 1 & & & & 1 & & \\
\hline & 6 & & & +4 & 10 & 71 & 46 & & +7 & 25 & \\
\hline & & 2 & & 5 & 15 & & & & & 5 & \\
\hline & & & 1 & +2 & 1 & & & & 1 & & \\
\hline & & & 2 & 1 & 20 & 52 & 2 & & 1 & 46 & \\
\hline \multirow{5}{*}{$\begin{array}{l}\text { ROCK DOVE } \\
\text { GREAT HORNED OWL } \\
\text { SNOWY OWL } \\
\text { DOWNY WOODPECKER } \\
\text { HAIRY WOODPECKER } \\
\end{array}$} & 28 & & & 25 & 6 & 85 & 33 & & & & \\
\hline & 1 & 1 & 1 & 2 & +1 & 1 & 1 & & 1 & 3 & \\
\hline & & & & +1 & & 2 & 2 & & & & \\
\hline & 1 & 1 & & 4 & 9 & 1 & & & 8 & & \\
\hline & & 1 & 1 & 1 & 9 & 1 & & +2 & 6 & & 1 \\
\hline \multirow{5}{*}{$\begin{array}{l}\text { THREE-TOED WOODPECKER } \\
\text { BLACK-BACKED WOODPECKER } \\
\text { NORTHERN FLICKER } \\
\text { PILEATED WOODPECKER } \\
\text { HORNED LARK }\end{array}$} & & & & & 1 & & & 2 & & & \\
\hline & & & & & & & & & & & \\
\hline & & & & & +1 & & & & & 1 & \\
\hline & & & & & & & & & +1 & & \\
\hline & & 21 & & & 6 & 6 & & & & 519 & \\
\hline \multirow{5}{*}{$\begin{array}{l}\text { GRAY JAY } \\
\text { BLUE JAY } \\
\text { BLACK-BILLED MAGPIE } \\
\text { COMMON RAVEN } \\
\text { BLACK-CAPPED CHICKADEE }\end{array}$} & & & & & & & & 2 & & & \\
\hline & & & 1 & 5 & 1 & & & 4 & 2 & & 3 \\
\hline & 11 & 75 & 6 & 20 & 168 & 95 & 3 & 6 & 10 & 4 & 12 \\
\hline & & & 14 & & 3 & & & 5 & 2 & & 37 \\
\hline & 3 & 9 & 7 & 33 & 65 & 4 & +2 & 4 & 30 & & 12 \\
\hline \multirow{5}{*}{$\begin{array}{l}\text { BOREAL CHICKADEE } \\
\text { RED-BREASTED NUTHATCH } \\
\text { WHITE-BREASTED NUTHATCH } \\
\text { AMERICAN ROBIN } \\
\text { BOHEMIAN WAXWING } \\
\end{array}$} & & & & & & & & & & & \\
\hline & & & & 2 & 4 & & & & & & \\
\hline & & & & 1 & & & & & 1 & & \\
\hline & & & & & & & & & & 1 & \\
\hline & 25 & 100 & & 17 & 54 & & & +30 & +3 & 2 & \\
\hline \multirow{5}{*}{$\begin{array}{l}\text { NORTHERN SHRIKE } \\
\text { EUROPEAN STARLING } \\
\text { AMERICAN TREE SPARROW } \\
\text { DARK-EYED JUNCO } \\
\text { SNOW BUNTING }\end{array}$} & & & & +1 & 2 & 1 & & & & & \\
\hline & & & & 3 & & & & & & 3 & \\
\hline & & & & & 6 & & & & & 1 & \\
\hline & & & 1 & & 2 & & & & & 1 & \\
\hline & 9 & & 150 & 100 & 222 & 3740 & 50 & 100 & 95 & 1147 & 469 \\
\hline \multirow{5}{*}{$\begin{array}{l}\text { RUSTY BLACKBIRD } \\
\text { ROSY FINCH } \\
\text { PINE GROSBEAK } \\
\text { RED CROSSBILL } \\
\text { WHITE-WINGED CROSSBILL }\end{array}$} & & & & & 17 & & & & & 1 & \\
\hline & & & & & 488 & & & & & 3 & \\
\hline & & & 15 & & 2 & & & +25 & 1 & & \\
\hline & & & & & 7 & & & & & & \\
\hline & & & & & & & & & & & \\
\hline \multirow{4}{*}{$\begin{array}{l}\text { COMMON REDPOLL } \\
\text { HOARY REDPOLL } \\
\text { EVENING GROSBEAK } \\
\text { HOUSE SPARROW }\end{array}$} & & 2 & & +2 & 41 & & & & & 7 & \\
\hline & & & & & & & & & & & \\
\hline & & & & 25 & & & & 50 & 8 & & 5 \\
\hline & 549 & 6 & 14 & 311 & 40 & 1075 & 50 & 4 & 197 & 605 & 50 \\
\hline \multirow{4}{*}{$\begin{array}{l}\text { NO. SPECIES IN TABLE } 3 \& 4 \\
\text { NO. COUNT DAY SPECIES } \\
\text { NO. COUNT PERIOD SPECIES } \\
\text { NO. COUNT DAY INDIVIDUALS }\end{array}$} & 0 & 0 & 0 & 1 & 10 & 2 & 0 & 0 & 0 & 3 & 0 \\
\hline & 9 & 11 & 12 & 19 & 36 & 19 & 8 & 9 & 15 & 21 & 8 \\
\hline & 9 & 11 & 13 & 25 & 40 & 19 & 9 & 12 & 18 & 21 & 8 \\
\hline & 633 & 219 & 213 & 568 & 1227 & 5522 & 187 & 177 & 364 & 2473 & 589 \\
\hline
\end{tabular}


Table 2-3. SPECIES RECORDED FROM MORE THAN THREE LOCALITIES. A PLUS SIGN INDICATES A HIGH COUNT FORA SPECIES SEEN DURING COUNT PERIOD BUT NOT ON COUNT DAY.

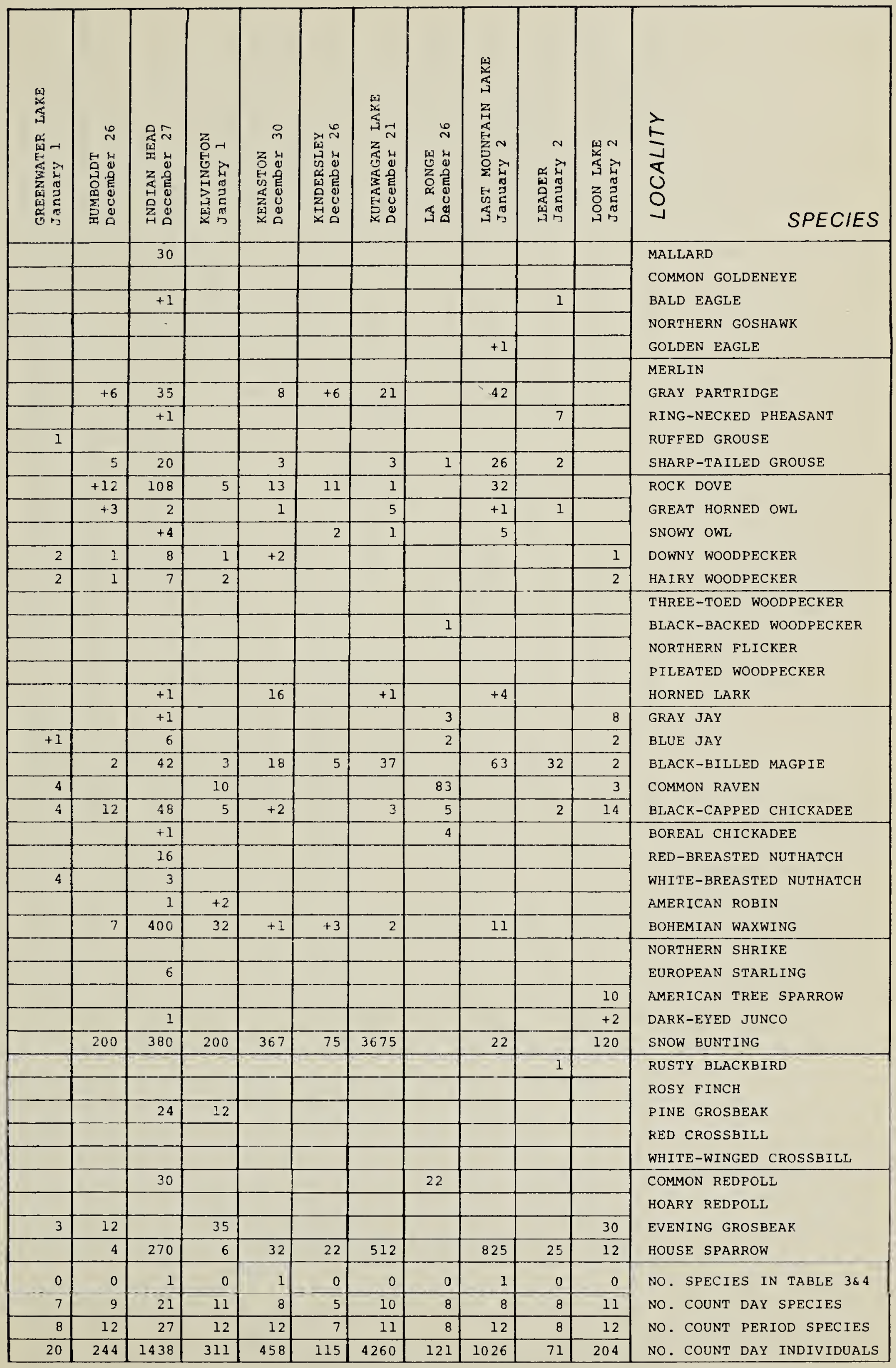


Table 2-4. SPECIES RECORDED FROM MORE THAN THREE LOCALITIES. A PLUS SIGN INDICATES A HIGH COUNT FOR A SPECIES SEEN DURING COUNT PERIOD BUT NOT ON COUNT DAY.

\section{SPECIES}
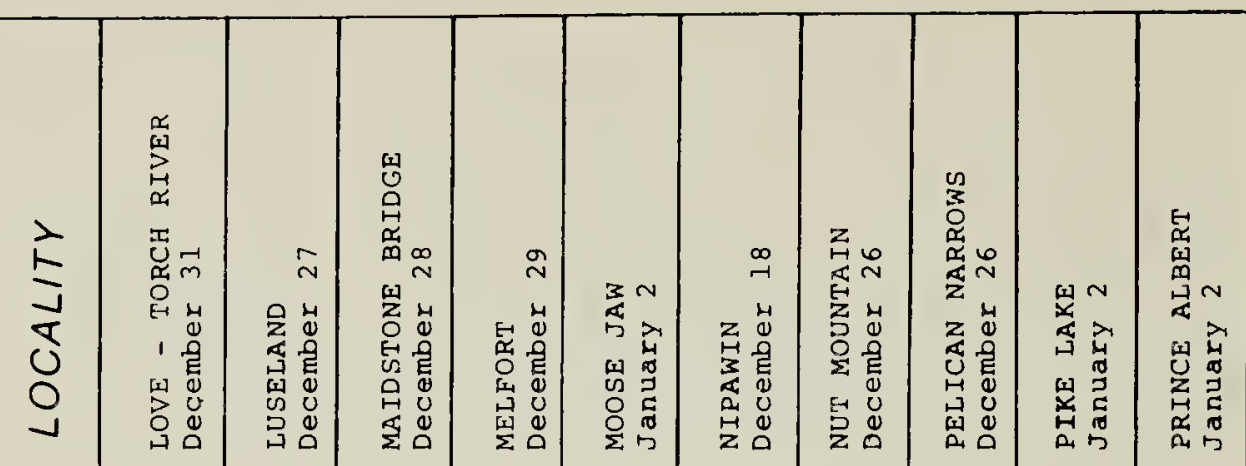

告

MALLARD

COMMON GOLDENEYE

BALD EAGLE

NORTHERN GOSHAWK

GOLDEN EAGLE

MERLIN

GRAY PARTRIDGE

RING-NECKED PHEASANT

RUFFED GROUSE

SHARP-TAILED GROUSE

ROCK DOVE

GREAT HORNED OWL

SNOWY OWL

DOWNY WOODPECKER

HAIRY WOODPECKER

THREE-TOED WOODPECKER

BLACK-BACKED WOODPECKER

NORTHERN FLICKER

PILEATED WOODPECKER

HORNED LARK

GRAY JAY

BLUE JAY

BLACK-BILLED MAGPIE

COMMON RAVEN

BLACK-CAPPED CHICKADEE

BOREAL CHICKADEE

RED-BREASTED NUTHATCH

WHITE-BREASTED NUTHATCH AMERICAN ROBIN

BOHEMIAN WAXWING

NORTHERN SHRIKE

EUROPEAN STARLING

AMERICAN TREE SPARROW

DARK-EYED JUNCO

SNOW BUNTING

RUSTY BLACKBIRD

ROSY FINCH

PINE GROSBEAK

RED CROSSBILL

WHITE-WINGED CROSSBILI

COMMON REDPOLL

HOARY REDPOLL

EVENING GROSBEAK

HOUSE SPARROW

NO. SPECIES IN TABLE $3 \& 4$

NO. COUNT DAY SPECIES

NO. COUNT PERIOD SPECIES

NO. COUNT DAY INDIVIDUALS

\begin{tabular}{|c|c|c|c|c|c|c|c|c|c|c|}
\hline & & 20 & $\Sigma 0$ & 20 & 40 & 40 & & $A n$ & 1417 & $\sim \Delta$ \\
\hline & & & & & & & & & & \\
\hline & & & & & & & & & & \\
\hline & & & & & & & & & & \\
\hline & & +1 & & & & & & 3 & & \\
\hline & & & & & & & & & & \\
\hline & 1 & & & +1 & & & & & & \\
\hline & 6 & +3 & & 105 & 6 & 6 & & & & \\
\hline & & & & 26 & & & & & & \\
\hline & & 1 & & & & 1 & & 2 & & 5 \\
\hline & & 17 & & 8 & & 1 & & 28 & & \\
\hline & 21 & 3 & +6 & 186 & 15 & & & 9 & & \\
\hline & & 1 & & & & & & 2 & & \\
\hline & & 1 & & 3 & +1 & 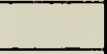 & & & & \\
\hline 1 & & 2 & 1 & 3 & & 3 & & 4 & 1 & 1 \\
\hline 6 & 1 & 3 & +1 & 1 & 2 & 2 & & 7 & 1 & 1 \\
\hline & & & & & & & +1 & & & 1 \\
\hline & & & & & & & 1 & & & \\
\hline & & & & & & & & & & \\
\hline & & & & & & & +1 & & & \\
\hline & 6 & +1 & & 3 & & & & & & \\
\hline 1 & & & & & 1 & 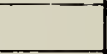 & +4 & & 1 & 9 \\
\hline 10 & 1 & 4 & & & 2 & 1 & & 16 & 4 & 1 \\
\hline 5 & 17 & 37 & 6 & 106 & 8 & 7 & & 82 & 2 & 8 \\
\hline 21 & & +2 & 2 & & 75 & +50 & 45 & 2 & 20 & 33 \\
\hline 32 & 2 & 36 & 12 & 28 & 3 & 7 & 4 & 47 & 10 & 19 \\
\hline 2 & & & 1 & & & & 1 & & & 21 \\
\hline & & & & +1 & & & & & & \\
\hline & & & & & & & & 2 & 1 & 2 \\
\hline & & & & 1 & & & & & & \\
\hline 4 & & & 42 & 544 & 24 & 25 & & 198 & & \\
\hline & 1 & 1 & & & & & & 4 & & \\
\hline & & & & 155 & 9 & & & & & \\
\hline & & & & & & & & & & \\
\hline & & & & & & & +2 & & & \\
\hline 151 & 2350 & 1320 & 50 & 85 & 11 & +150 & & 245 & 30 & \\
\hline & & & & & & & & & & \\
\hline & & & & & & & & & & \\
\hline+1 & & 4 & 6 & 8 & 18 & 2 & & 11 & & +2 \\
\hline & & & & & & & & & & \\
\hline & & & & & & & & & & \\
\hline & & & & & & & 15 & & & 62 \\
\hline & & & & & & & 10 & & & \\
\hline 130 & & 7 & 21 & 3 & 132 & 28 & & 31 & 30 & +30 \\
\hline 37 & 41 & 375 & 68 & 733 & 64 & & & 164 & & 1 \\
\hline 0 & 1 & 1 & 0 & 1 & 0 & 0 & 3 & 1 & 0 & 1 \\
\hline 12 & 12 & 16 & 10 & 18 & 14 & 11 & 6 & 19 & 10 & 14 \\
\hline 13 & 12 & 20 & 12 & 20 & 15 & 13 & 13 & 19 & 10 & 16 \\
\hline 400 & 2453 & 1837 & 209 & 1999 & 370 & 83 & 76 & 859 & 100 & 166 \\
\hline
\end{tabular}


Table 2-5. SPECIES RECORDED FROM MORE THAN THREE LOCALITIES. A PLUS SIGN INDICATES A HIGH COUNT FORA SPECIES SEEN DURING COUNT PERIOD BUT NOT ON COUNT DAY.

\begin{tabular}{|c|c|c|c|c|c|c|c|c|c|c|c|}
\hline 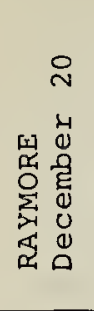 & 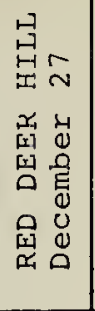 & 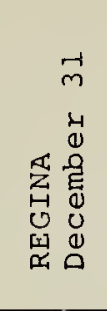 & 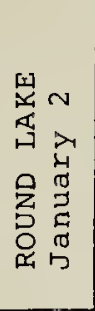 & 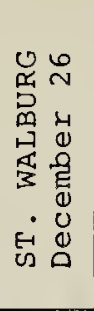 & 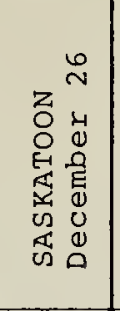 & 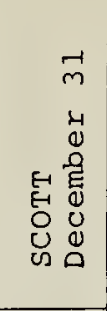 & 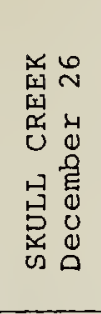 & 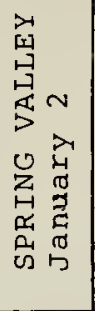 & 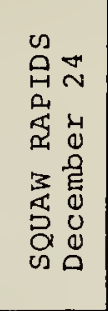 & 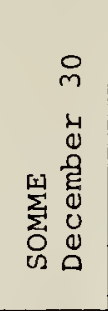 & $\begin{array}{l}\lambda \\
\frac{1}{1} \\
0 \\
0 \\
0\end{array}$ \\
\hline & & 10 & 13 & & 7 & & & & 6 & & \multirow{5}{*}{$\begin{array}{l}\text { MALLARD } \\
\text { COMMON GOLDENEYE } \\
\text { BALD EAGLE } \\
\text { NORTHERN GOSHAHK } \\
\text { GOLDEN EAGLE }\end{array}$} \\
\hline & & & & & 75 & & & & 56 & & \\
\hline 1 & & & & & & & & & 5 & & \\
\hline \multirow[t]{2}{*}{2} & & & & & & & & & 1 & +1 & \\
\hline & & & & & & & +2 & +1 & & & \\
\hline+1 & 1 & 3 & 1 & & 7 & & & & & & \multirow{5}{*}{$\begin{array}{l}\text { MERLIN } \\
\text { GRAY PARTRIDGE } \\
\text { RING-NECKED PHEASANT } \\
\text { RUFFED GROUSE } \\
\text { SHARP-TAILED GROUSE }\end{array}$} \\
\hline 19 & & 43 & 7 & & 29 & 42 & & 49 & & 8 & \\
\hline+1 & & & 6 & & & & 11 & & & & \\
\hline 4 & & 1 & 2 & 1 & & & & & 5 & 2 & \\
\hline 28 & 9 & 4 & 12 & 7 & 48 & 8 & +4 & 89 & 2 & 6 & \\
\hline 122 & 59 & 1039 & & & 1596 & 151 & & 24 & & +3 & \multirow{5}{*}{$\begin{array}{l}\text { ROCK DOVE } \\
\text { GREAT HORNED OWL } \\
\text { SNOWY OWL } \\
\text { DOWNY WOODPECKER } \\
\text { HAIRY WOODPECKER }\end{array}$} \\
\hline 5 & & 10 & 1 & & 4 & 2 & 2 & 2 & 1 & +1 & \\
\hline+1 & & 5 & & & 1 & 3 & & +1 & & 1 & \\
\hline 2 & & 2 & 9 & & 9 & & 4 & & 1 & 8 & \\
\hline \multirow[t]{5}{*}{3} & 1 & & 8 & 2 & 15 & & 1 & & 3 & 21 & \\
\hline & & & & & & & & & 2 & & \multirow{5}{*}{$\begin{array}{l}\text { THREE-TOED WOODPECKER } \\
\text { BLACK-BACKED WOODPECKER } \\
\text { NORTHERN FLICKER } \\
\text { PILEATED WOODPECKER }\end{array}$} \\
\hline & & & & & & 1 & & & 1 & & \\
\hline & & & & & 1 & & 1 & & $\ldots$ & & \\
\hline & & & 2 & & & & & & 1 & 3 & \\
\hline \multirow[t]{2}{*}{5} & & 1 & & & & 1 & 56 & 7 & & & \\
\hline & 11 & & & 1 & & & & & 3 & 14 & \multirow{5}{*}{$\begin{array}{l}\text { GRAY JAY } \\
\text { BLUE JAY } \\
\text { BLACK-BILLED MAGPIE } \\
\text { COMMON RAVEN } \\
\text { BLACK-CAPPED CHICKADEE }\end{array}$} \\
\hline+1 & 4 & & 5 & 2 & 23 & & 1 & & 3 & 20 & \\
\hline 53 & 26 & 45 & 25 & 5 & 424 & 34 & 16 & 12 & 6 & 23 & \\
\hline 1 & 32 & & 1 & 12 & & 1 & & & 69 & 61 & \\
\hline \multirow[t]{5}{*}{34} & 26 & 25 & 35 & 7 & 130 & 6 & 15 & & 3 & 57 & \\
\hline & & 4 & $L$ & 6 & 9 & 4 & & & 8 & 3 & \multirow{4}{*}{$\begin{array}{l}\text { BOREAL CHICKADEE } \\
\text { RED-BREASTED NUTHATCH } \\
\text { WHITE-BREASTED NUTHATCH } \\
\text { AMERICAN ROBIN }\end{array}$} \\
\hline & & 6 & & & 18 & & & & 1 & & \\
\hline & & 2 & 10 & & 1 & & & & & 1 & \\
\hline & & & & & 1 & & & & & & \\
\hline 17 & 13 & 934 & & 6 & 6047 & 3 & +110 & 47 & & +5 & BOHEMIAN WAXWING \\
\hline 2 & & & +1 & & & & +1 & & & 1 & \multirow{5}{*}{$\begin{array}{l}\text { NORTHERN SHRIKE } \\
\text { EUROPEAN · STARLING } \\
\text { AMERICAN TREE SPARROW } \\
\text { DARK-EYED JUNCO } \\
\text { SNOW BUNTING }\end{array}$} \\
\hline \multirow[t]{2}{*}{3} & & & 6 & 6 & 1000 & & & & & & \\
\hline & & & & & & & 6 & & & & \\
\hline 1 & & 1 & & & & & & & & & \\
\hline 2762 & 185 & 496 & 250 & 225 & 921 & 2817 & 15 & 113 & 895 & 753 & \\
\hline \multirow[t]{2}{*}{1} & & & +1 & & & & & & & & \multirow{2}{*}{$\begin{array}{l}\text { RUSTY BLACKBIRD } \\
\text { ROSY FINCH }\end{array}$} \\
\hline & & & & & & & & & & & \\
\hline \multirow[t]{2}{*}{6} & 81 & 1 & & 4 & 12 & 10 & & & 9 & 35 & \multirow{3}{*}{$\begin{array}{l}\text { PINE GROSBEAK } \\
\text { RED CROSSBILL } \\
\text { WHITE-WINGED CROSSBILL }\end{array}$} \\
\hline & 32 & & & & 2 & & & & 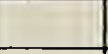 & & \\
\hline 2 & & 10 & & & & 5 & & & & & \\
\hline 217 & & & & & 18 & & & & 160 & & \multirow{4}{*}{$\begin{array}{l}\text { COMMON REDPOLL } \\
\text { HOARY REDPOLL } \\
\text { EVENING GROSBEAK } \\
\text { HOUSE SPARROW } \\
\end{array}$} \\
\hline 2 & & & & & & & & & 1 & & \\
\hline 3 & 25 & & 50 & & 44 & & & & 8 & 83 & \\
\hline 1437 & 40 & 1625 & 40 & 7 & 2957 & 307 & 35 & 360 & & 46 & \\
\hline 0 & 0 & 4 & 1 & 0 & 6 & 0 & 2 & 0 & 4 & 1 & \multirow{4}{*}{$\begin{array}{l}\text { NO. SPECIES IN TABLE } 3 \& 4 \\
\text { NO. COUNT DAY SPECIES } \\
\text { NO. COUNT PERIOD SPECIES } \\
\text { NO. COUNT DAY INDIVIDUALS }\end{array}$} \\
\hline 25 & 15 & 25 & 19 & 14 & 30 & 16 & 13 & 9 & 26 & 20 & \\
\hline 29 & 15 & 25 & 22 & 14 & 32 & 16 & 18 & 11 & 26 & 24 & \\
\hline 4732 & 545 & 4834 & 483 & 291 & 13421 & 3395 & 172 & 703 & 1397 & 1147 & \\
\hline
\end{tabular}


Table 2-6. SPECIES RECORDED FROM MORE THAN THREE LOCALITIES. A PLUS SIGN INDICATES A HIGH COUNT FORA SPECIES SEEN DURING COUNT PERIOD BUT NOT ON COUNT DAY.

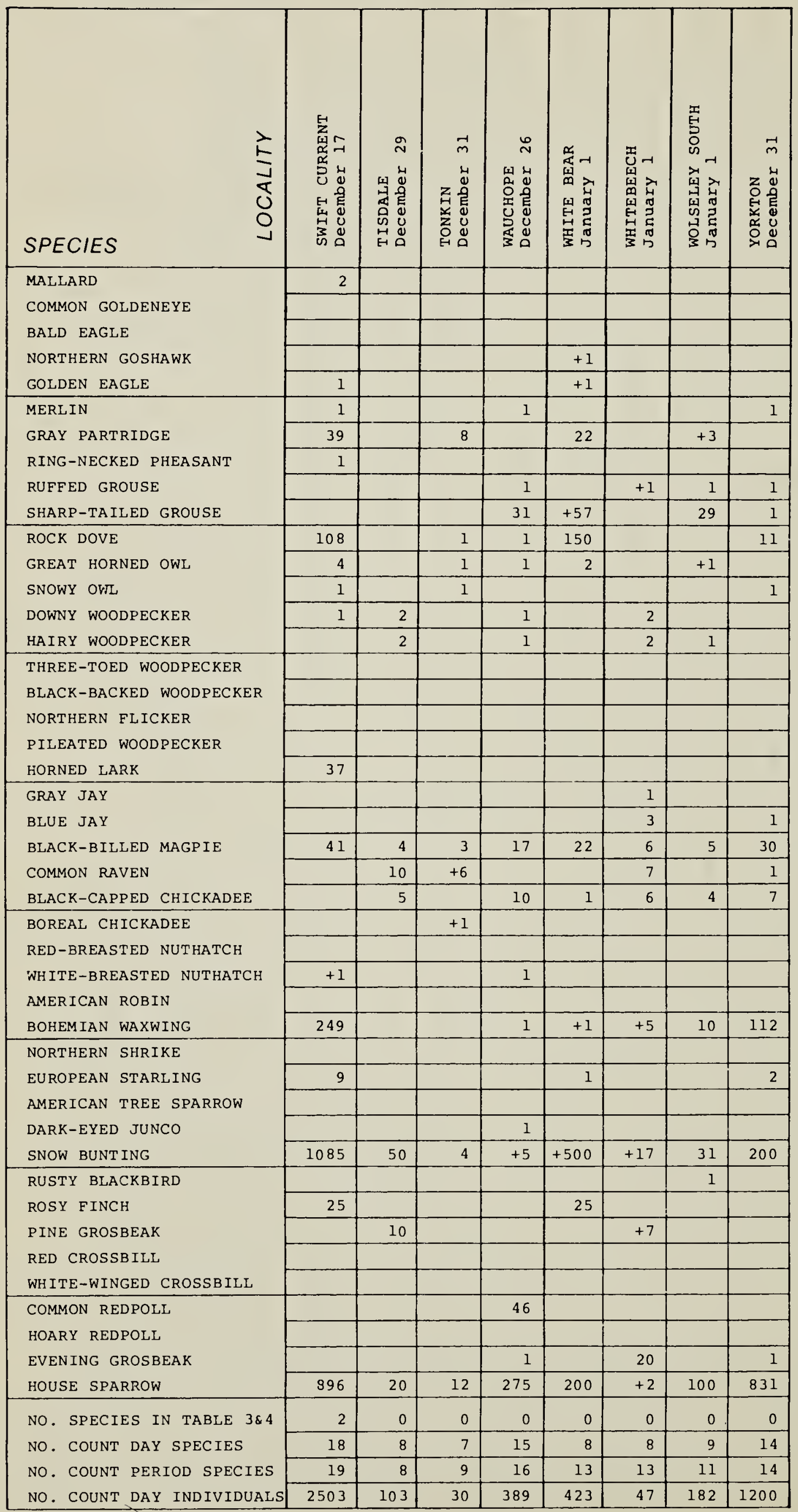


Table 3. SPECIES SEEN IN THREE OR FEWER LOCALITIES.

\begin{tabular}{|c|c|}
\hline SPECIES & LOCALITIES AND NUMBERS SEEN \\
\hline \multirow{5}{*}{$\begin{array}{l}\text { COMMON LOON } \\
\text { HORNED GREBE } \\
\text { CANADA GOOSE } \\
\text { COMMON MERGANSER } \\
\text { ROUGH-LEGGED HAWK }\end{array}$} & GARDINER DAM， 1 \\
\hline & SASKATOON, 1 \\
\hline & REGINA， 550 \\
\hline & GARDINER DAM， 27; SQUAW RAPIDS, 9 \\
\hline & FORT WALSH, + 1 \\
\hline \multirow{5}{*}{$\begin{array}{l}\text { GYRFALCON } \\
\text { PRAIRIE FALCON } \\
\text { SPRUCE GROUSE } \\
\text { SAGE GROUSE } \\
\text { WILD TURKEY }\end{array}$} & BIGGAR, +1 ; BIG GULLY CREEK, 1 \\
\hline & INDIAN HEAD, 1 ; SKULL CREEK, + 2 \\
\hline & P.A. NATIONAL PARK, 2 \\
\hline & FORT WALSH, 1; GOVENLOCK, 95 \\
\hline & FORT WALSH, 1 \\
\hline \multirow{5}{*}{$\begin{array}{l}\text { COMMON SNIPE } \\
\text { NORTHERN HAWK-OWL } \\
\text { BARRED OWL } \\
\text { SHORT-EARED OWL } \\
\text { BOREAL OWL }\end{array}$} & BIG GULLY CREEK, 1 \\
\hline & PELICAN NARROWS, +1 \\
\hline & SOMME, 1 \\
\hline & GOVENLOCK, 1 ; LAST MOUNTAIN LAKE, +1 ; MOOSE JAW, 1 \\
\hline & SQUAW RAPIDS, 1 \\
\hline \multirow{5}{*}{$\begin{array}{l}\text { BELTED KINGFISHER } \\
\text { AMERICAN CROW } \\
\text { BROWN CREEPER } \\
\text { GOLDEN-CROWNED KINGLET } \\
\text { TOWNSEND'S SOLITAIRE }\end{array}$} & FORT WALSH, 1 \\
\hline & REGINA， 1 \\
\hline & FORT WALSH, 1 ; ROUND LAKE, +1 ; SWIFT CURRENT, 1 \\
\hline & FORT WALSH, 2; PIKE LAKE, 2 \\
\hline & SASKATOON, +1 \\
\hline \multirow{6}{*}{$\begin{array}{l}\text { CEDAR WAXWING } \\
\text { SONG SPARROW } \\
\text { WHITE-THROATED SPARROW } \\
\text { HARRIS' SPARROW } \\
\text { RED-WINGED BLACKBIRD } \\
\text { PURPLE FINCH }\end{array}$} & LUSELAND， 6 ; SASKATOON, 19 \\
\hline & KENASTON, +1 \\
\hline & REGINA， 1 ; SASKATOON, 1 \\
\hline & SASKATOON, 1 \\
\hline & FORT WALSH, 1 \\
\hline & FORT QU'APPELLE, 2 ; SASKATOON, +1; SWIFT CURRENT, 2 \\
\hline
\end{tabular}

\section{Table 4. BIRDS NOT IDENTIFIED TO SPECIES}

\begin{tabular}{|c|c|}
\hline SPECIES GROUP & LOCALITIES AND NUMBERS SEEN \\
\hline \multirow{4}{*}{$\begin{array}{l}\text { GROUSE species } \\
\text { WOODPECKER species } \\
\text { LONGSPUR species } \\
\text { BLACKBIRD species }\end{array}$} & PELICAN NARROWS, +3 \\
\hline & BIG GULLY CREEK, 1 ; SQUAW RAPIDS, 3 \\
\hline & GOVENLOCK, $1 ;$ PELICAN NARROWS, +7 \\
\hline & FORT WALSH, 1 \\
\hline \multirow{4}{*}{$\begin{array}{l}\text { CARPODACUS (Purple or Cassin's Finch) } \\
\text { GROSBEAK - FINCH species } \\
\text { CROSSBILL species } \\
\text { REDPOLL species }\end{array}$} & FORT WALSH, 1 \\
\hline & SKULL CREEK, 9 \\
\hline & FORT WALSH, 9; REGINA, 15 \\
\hline & BIG GULLY CREFK, 35; MAIDSTONE BRIDGE, 25; SQUAW RAPIDS, 134 \\
\hline
\end{tabular}

